\title{
A new semi-automatic approach to find suitable virtual electrodes in arrays using an interpolation strategy
}

\author{
Christina Salchow, Markus Valtin, Thomas Seel, Thomas Schauer \\ Control Systems Group, Technische Universität Berlin, Berlin, Germany \\ This article is distributed under the terms of the Creative Commons Attribution Noncommercial License (CC BY-NC 4.0) which \\ permits any noncommercial use, distribution, and reproduction in any medium, provided the original author(s) and source are credited.
}

\begin{abstract}
Functional Electrical Stimulation via electrode arrays enables the user to form virtual electrodes (VEs) of dynamic shape, size, and position. We developed a feedback-controlassisted manual search strategy which allows the therapist to conveniently and continuously modify VEs to find a good stimulation area. This works for applications in which the desired movement consists of at least two degrees of freedom. The virtual electrode can be moved to arbitrary locations within the array, and each involved element is stimulated with an individual intensity. Meanwhile, the applied global stimulation intensity is controlled automatically to meet a predefined angle for one degree of freedom. This enables the therapist to concentrate on the remaining degree(s) of freedom while changing the VE position. This feedback-controlassisted approach aims to integrate the user's opinion and the patient's sensation. Therefore, our method bridges the gap between manual search and fully automatic identification procedures for array electrodes. Measurements in four healthy volunteers were performed to demonstrate the usefulness of our concept, using a 24-element array to generate wrist and hand extension.
\end{abstract}

Key Words: functional electrical stimulation, electrode arrays, virtual electrode, identification strategy, feedback control, hand extension

Eur J Transl Myol 201626 (2) 134-139

Functional Electrical Stimulation (FES) is a widely used technique for physical rehabilitation of patients after stroke or spinal cord injury. Its application via surface electrodes has several disadvantages such as long placement times and static electrode positions during therapy sessions. Electrode arrays (or multi-pad electrodes) help to overcome these problems and have become popular in FES research within the last decade. ${ }^{1}$ Electrode arrays consist of multiple, small elements, which can be activated separately. Virtual electrodes (VEs) can be formed by any combination of those elements and can dynamically change position and size. This allows for repositioning of the (virtual) stimulation electrode by simply choosing different subsets of active elements.

A manual search for VEs within arrays is laborious and time consuming, because the level of stimulation has to be adjusted for each tested VE. Many approaches have been introduced to automatically find the optimal stimulation point(s) for defined movements within an array. Automatic search algorithms require the formulation of a selection criterion, or cost function. Most procedures apply a motion-based criterion and compare the twitch or step responses registered for each element. Such a criterion can be the fit with a reference trajectory, which is derived from the movement of healthy people, the achievement of predefined angular constraints, or the maximum registered movement amplitud together with additional constraints. ${ }^{2-5}$ Those algorithms do not take into account the individual opinion of a therapist and the patient's sensing. This may lead to poor acceptance in clinical practice. Furthermore, the search is restricted, as the VE is formed by a subset of elements which get the same stimulation intensity.

We introduce a feedback-control-assisted approach that is faster and more convenient than manual search and overcomes the lack of user integration and acceptance of fully automatic identification procedures. It enables the therapist to conveniently manipulate a VE within an array for a desired motion. In the presented framework, the center of a VE can be modified fluently to arbitrary positions within the array. Different shapes and sizes are possible. This is achieved by the following two components: i) An interpolation function automatically determines to what extent elements are involved in the current VE and calculates individual stimulation intensities for those elements; ii) 


\section{Finding suitable virtual electrodes in arrays using an interpolation strategy}

Eur J Transl Myol 26 (2) 134-139

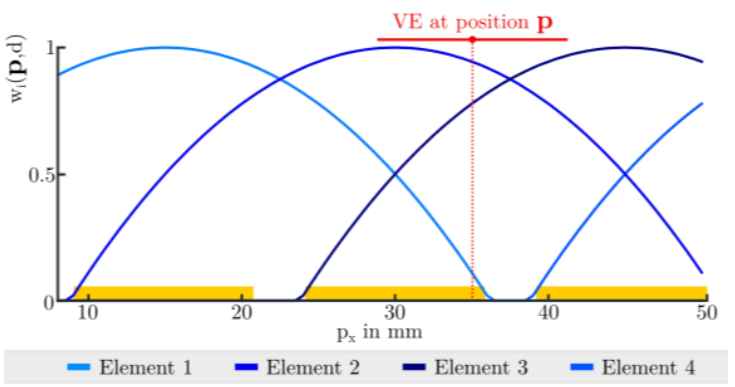

Fig 1. One-dimensional weight function $w_{i}(\boldsymbol{p}, d)$ of four neighboring elements for the virtual positions $\boldsymbol{p}=\left[8 . .50, p_{y}\right]$, where $p_{y}$ is a fixed value. The elements of $12 \mathrm{~mm}$ width are marked in yellow (1,2, and 3). The red line marks the size of an exemplary VE of $d=12 \mathrm{~mm}$ with the center at $\boldsymbol{p}=\left[35, p_{y}\right]$.

The applied global stimulation intensity is constantly adjusted by the system, so that a predefined angle in one degree of freedom (DoF) is achieved.

For example, in a drop foot experiment, the dorsiflexion of the foot would be controlled to meet a desired angle, while the therapist can modify the VE and observe the reaction of different sizes and positions of VEs to balance eversion and inversion of the foot. ${ }^{6}$ With our approach, the therapist has the chance to find individual stimulation areas according to a patient's needs and personal training strategy.

We will explain the method in detail in the following section. In the experimental validation, we evaluate the feasibility of our concept in an experiment with an electrode array on the forearm, which is used to generate hand opening in four healthy volunteers.

\section{Materials and Methods}

\section{The Interpolation Problem}

The standard approach for finding suitable VEs in electrode arrays is to observe the motion that is caused by applying stimulation to discrete positions, i. e. the elements are either deactivated or stimulated at the same (global) stimulation intensity. We aimed to overcome the restriction of discrete VE positions by providing a smooth interpolation function for the area of the array. Therefore, we consider the following: We have an array with a matrix of $n$ elements, which covers a defined two dimensional area. Within this area, the center point $\boldsymbol{p} \in \mathbb{R}^{2}$ of a VE can be placed arbitrarily. The VE model is circular and parametrized by a diameter $d$. The global stimulation intensity $u$ is applied to the VE. Each array element is assigned an individual stimulation intensity $q_{i}=1 \ldots n$, which is related to the global intensity $u$ and the VE via an interpolation function $f_{i}(u, \boldsymbol{p}, d)$. This function determines whether an element should be activated and which individual intensity $q_{i}$ is applied depending on the position $\boldsymbol{p}$ and diameter $d$ of the virtual electrode. We employed a quadratic relationship as a weight function $w_{i}(\boldsymbol{p}, d)(1)$. The resulting virtual electrode consists of active elements within the area of the VE model with individual stimulation intensities according to the distance of the element to the center $\boldsymbol{p}$.

$$
\begin{aligned}
w_{i}(\boldsymbol{p}, d) & =\frac{1}{\sigma(d)} \cdot\left(\sigma(d)-\left\|\boldsymbol{p}_{i}-\boldsymbol{p}\right\|_{2}^{2}\right) \\
f_{i}(u, \boldsymbol{p}, d) & =\left\{\begin{aligned}
w_{i}(\boldsymbol{p}, d) \cdot u, & \text { if } w_{i}(\boldsymbol{p}, d)>0 \\
0, & \text { otherwise. }
\end{aligned}\right.
\end{aligned}
$$

Here $\boldsymbol{p}_{\boldsymbol{i}} \in \mathbb{R}^{2}$ marks the position vector for the center of the $i^{\text {th }}$ element. $\sigma(d)$ establishes the function radius depending on the VE diameter $(\sigma(d) \approx 30 \cdot d)$ and was determined empirically. Figure 1 exemplifies the quadratic weight functions of four neighboring elements in one dimension. In this example, the VE partly covers element 2 and element 3 . As a result, both elements account for the VE and achieve high stimulation intensities $q_{i}$ according to their weight function values close to 1 .

\section{Controller Design}

Consider the task of finding suitable VEs for a specific motion with more than one DoF, e. g. hand opening. Define one of these DoF as the major DoF, e. g. wrist extension, and the other one (or more) as minor DoF. The standard manual approach for testing different elements in electrode arrays is to choose an element, increase the stimulation intensity until a certain degree of motion of the major DoF is achieved, observe the resulting motion of the minor DoF, and repeat this procedure for the next element.

To overcome the laboriousness of this testing procedure, we propose an automatic adjustment of the global stimulation intensity $u$. The goal was to relieve the therapist from tuning the intensity manually at each tested position. Our system constantly controls the global intensity $u$, such that a predefined angle $r$ is achieved in the major DoF $y$, e.g. wrist extension. The remaining DoF $\boldsymbol{y}^{\prime}$ can be observed by the user, who is able to influence the generated motion by changing size and position of the VE. Fig. 2 illustrates the system design of our feedback-control-assisted strategy.

Finding a controller $K$, that adjusts the stimulation intensity robustly throughout the whole electrode array, is a challenge, because the stimulation response varies strongly between single elements. Therefore, we quantified the behavior throughout the array by identifying a first-order model (3) with delay for each element.

$$
G_{i}(s)=\frac{k_{i}}{T_{i} \cdot s+1} \cdot e^{T} d i \cdot s
$$

Step responses of $2 \mathrm{~s}$ were recorded for every element. The stimulation intensity started at motor threshold level $u_{\mathrm{m}}$ and jumped up to a predefined step stimulation level $u_{\text {step }} . u_{\mathrm{m}}$ was estimated from one single element as the highest stimulation intensity which still has not caused a movement. At the same element, $u_{\text {step }}$ was 


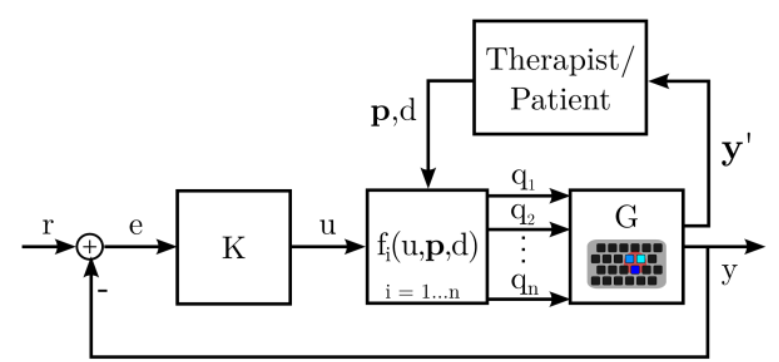

Fig 2. Overview of the closed-loop system. A controller $K$ adjusts the global intensity $u$ based on the error $e$ between the angular output of one DoF y and the reference angle $r$. The interpolation function $f_{i}(u, p, d)$ assigns an individual intensity $q_{i}$ to the elements in the plant $G$, which consists of the FES system, the array, the patient, and a motion measurement system. The motion in the remaining DoF y' can be observed by the therapist/patient who can adjust the position $p$ and diameter $d$ of the $V E$.

estimated as the intensity leading to a significant, not saturated movement. The data of each step was offsetremoved before the model was fitted with MATLAB's System Identification toolbox. The identified parameters were averaged, and then used to adjust the controller parameters. We decided to use a PID controller in its parallel form (4). The controller parameters were adjusted according to the Chien, Hrones and Reswick set-point method. ${ }^{7}$ Furthermore, a dead-band of $\pm 1^{\circ}$ was chosen for the PID controller. An anti-wind up was realized with a gain of 1 .

$$
\begin{aligned}
K(s) & =P+I \cdot \frac{1}{s}+D \cdot \frac{N \cdot s}{s+N} \\
P & =0.6 \cdot \frac{k}{T \cdot T_{d}} \\
I & =P \cdot \frac{1}{T} \\
D & =P \cdot \frac{T_{d}}{2}
\end{aligned}
$$

\section{Experimental Setup}

We evaluated the feasibility of our method with an electrode array on the forearm to generate hand opening. Here, the dominating DoF $y$ is a straight wrist extension $\alpha$. Usually, ulnar and radial wrist abduction $\beta$ shall be prevented and represent the remaining DoF $\boldsymbol{y}^{\prime}$ together with finger extension. In the experiments, we investigated whether our controller $K$ is able to track a desired wrist extension $y$ through the array and whether our approach allows for finding suitable VEs for hand opening.

FES was applied via the slightly modified DeltaStim System (HASOMED GmbH, Germany). ${ }^{8}$ We used a customized electrode array of 24 elements of a square shape $(12 \times 12 \mathrm{~mm})$ as shown in Fig. 3, with a single hydro-gel layer (AG702 Stimulating Gel, Axelgaard Manufacturing Co., Ltd., USA). ${ }^{6}$ The electrode was placed over the hand and finger extensors in the upper half of the forearm. A common counter electrode was placed at approximately $1 \mathrm{~cm}$ distance in distal direction. The global intensity $u$ equals the normalized charge of the stimulation pulses. The charge itself is defined as the product of the current amplitude $I$ and pulse width $p w(u=0: I=0 \mathrm{~mA}, p w=10 \mu \mathrm{s} ; u=1$ : $I=50 \mathrm{~mA}, p w=500 \mu \mathrm{s})$. In our setup, both quantities have been increased or decreased simultaneously while remaining a constant ratio (see Shalaby 2011 for details). ${ }^{9}$ The stimulation frequency was $25 \mathrm{~Hz}$. Our system has the ability to apply up to ten different biphasic stimulation pulses asynchronously to the active elements, which form the VE. Two inertial measurement units (MTx, Xsens Technologies B.V., Netherlands), one placed on the forearm and one on the back of the hand, were used to track the wrist extension (positive) and flexion (negative) angle $\alpha(=y)$, and the wrist abduction angle $\beta\left(=y^{\prime}\right)$. The experiments started with the hand and forearm lying flat on a table $(\alpha=0)$. Measurements were performed on four healthy volunteers ( $1 \mathrm{~F}, 3 \mathrm{M}$, age $31 \pm 9$ years). They were instructed to keep their forearm muscles relaxed and to not watch their own hand motion during testing. The controller design and implementation were performed in MATLAB/Simulink.

Instead of having a therapist adjusting the VE position $\boldsymbol{p}$, we defined paths for $\boldsymbol{p}$ through the whole array and VEs of different sizes. This allowed to compare the results of all volunteers and to prove the desired functioning of our framework. Those paths are illustrated in Fig. 3. In Experiment 1), a small VE of $d=12 \mathrm{~mm}$, which equals the size of a single element, started with $\boldsymbol{p}$ at the center of element 1 and was shifted from one element to the next through the whole array (see orange arrows in Fig. 3.a). The center $\boldsymbol{p}$ stayed for $2.5 \mathrm{~s}$ on each element and was then shifted within $5 \mathrm{~s}$ to the neighboring element with a stop of $2.5 \mathrm{~s}$ between the neighboring elements. This allowed for closer determination of the effect of the interpolation function. Experiment 2) was performed using a VE of $d=30 \mathrm{~mm}$, which approximately covered three elements (Fig. 3.b, light blue circles). The center $\boldsymbol{p}$ was shifted along the blue arrows with stops of $2.5 \mathrm{~s}$ at every arrowhead. Each experiment was performed at least twice: first in open-loop with a constant $u$ and afterwards in closed-loop (cf. Fig. 2).

\section{Results}

For the first-order model with delay, we achieved average data fits of $81-94 \%$. The identified parameters of all volunteers are summarized in Tab. 1. The results proved that the stimulation response varies strongly between single elements. We observed large variations in $k_{i}$ for the different elements as well as the different volunteers. Further, we had to deal with long 


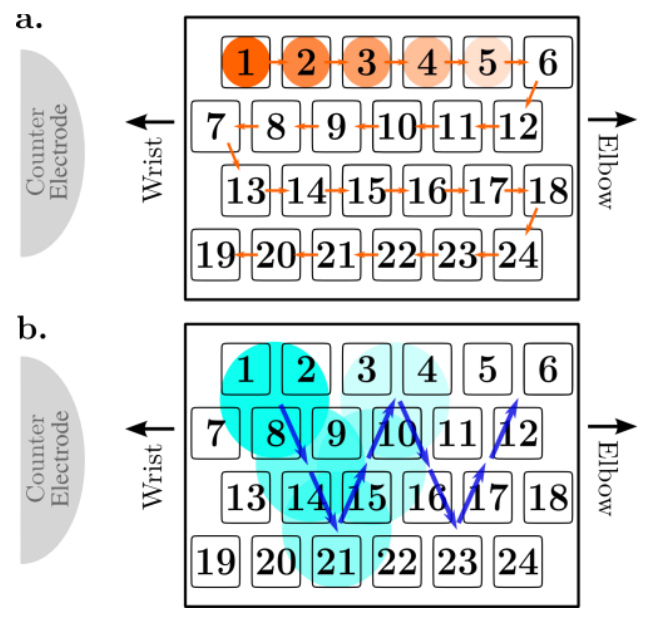

Fig 3. Custom-made 24-element array with routes for Experiment 1 (a.) and Experiment 2 (b.). The colored circles and arrows mark the positions $\boldsymbol{p}$ of the VE that were sequentially tested within the experiments. In $a$. the stimulated VE ( $d=12 \mathrm{~mm}$, orange) started at element 1 and then slowly moved to element 2, then to element 3 and so on. The sequence is highlighted by a color gradient. In $\boldsymbol{b}$. the stimulated VE of $d=30 \mathrm{~mm}$ (blue) started with its center between the elements 1,2 , and 8 .

delays of up to $300 \mathrm{~ms}$. Recording step responses for each array element, and identifying a model for each one was time consuming. We analyzed if we could speed up the process through using only five elements, which were spread across the array. That worked out well: $k_{\text {avg, } 5}$ and $k_{\text {avg }}$ were similar (Tab. 1). Hence, we used the averaged parameters from those five elements for calculating the controller parameters.

Fig. 4 displays the results of Experiment 1) of volunteer no. 1 in open-loop (a.) and closed-loop (b.) mode. For a varying $\boldsymbol{p}$, different reactions in both wrist angles were observed for a constant stimulation intensity. In closedloop, a steady wrist extension $\alpha$ was achieved close to $r=20^{\circ}$ for all tested positions. For all volunteers, the root mean squared errors between reference and measured angle $\alpha$ for Experiment 1) constituted $2^{\circ}$ to $8^{\circ}$. The largest errors were found during the shift of $\boldsymbol{p}$, marked as gray areas in Fig. 4. This was expected as for the controller the movement and therefore the changing charge distribution appears as a disturbance. From the course of the actuating variable $u$, areas with a high $(20-40 \mathrm{~s})$ and a low gain $(60-80 \mathrm{~s})$ for wrist extension can be identified. Two areas were identified where the wrist abduction $\beta$ was close to zero and still a sufficient wrist extension $\alpha$ was achieved (see Fig. 4.b at 30-40 s, where element 6, 11, and 12 was stimulated, and 90-120 s with element 14-17).
Table 1. Identified system parameters for every volunteer. $k_{\min }, k_{\max }$, and $k_{\text {avg }}$ refer to the minimal, maximal and average of the gain over all identified elements $i=1 \ldots 24 . k_{\text {avg, } 5}$ is the average for $i=(2,5,9,17,20) . T_{a v g}$ and $T_{d, a v g}$ are the averaged time constant and delay in seconds over all identified elements.

\begin{tabular}{lcccccc}
\hline Vol. & $k_{\min }$ & $k_{\max }$ & $k_{\text {avg }}$ & $k_{\text {avg, } 5}$ & $T_{\text {avg }}$ & $T_{\text {d,avg }}$ \\
\hline $\mathbf{1}$ & 204 & 570 & 405 & 404 & 0.20 & 0.15 \\
$\mathbf{2}$ & 730 & 1226 & 951 & 995 & 0.23 & 0.19 \\
$\mathbf{3}$ & 40 & 491 & 206 & 215 & 0.09 & 0.23 \\
$\mathbf{4}$ & 34 & 503 & 302 & 408 & 0.21 & 0.24 \\
\hline
\end{tabular}

Fig. 5 displays the results of Experiment 2) for the same volunteer. Compared to the application of the smaller VE in Experiment 1), the wrist angles showed less variation for both approaches, the open and the closed-loop. This resulted from more than one element being active at each tested position in the path. For $r=20^{\circ}$, the root mean squared errors between reference and measured angle $\alpha$ constituted $2.7^{\circ}$ to $4.8^{\circ}$ for the four volunteers. Two good VEs were identified with a wrist abduction $\beta$ close to zero (see Fig. 5.b at $\approx 35 \mathrm{~s}$ and $\approx 40 \mathrm{~s}$ ).

\section{Discussion}

The results demonstrated that the intensity, required for the predefined wrist extension, varies for the different positions in the array. The implemented PID controller was able to adjust to the different conditions after a certain period of time $(\approx 1 \mathrm{~s})$. Because the modification of $\boldsymbol{p}$ appeared as a disturbance, perfect reference tracking was not accomplished during the shift of the VE. Nevertheless, the achieved level of automatic adaption of the stimulation intensity will enable the therapist to search manually for a sufficient stimulation area, while completely focusing on the current hand posture. The results highlight that continuous update of the intensity is necessary to get a good sense about aband adduction of the wrist $(\beta)$ at the current position. The results are limited in their clinical application, as the experiments were simplified and were conducted with healthy humans only. The next step will be to test our framework with therapists and patients.

Our implementation allowed to employ all positions of the array through interpolation. The actual distribution of the stimulation charge was not yet determined, but it is certain that the actual area of stimulation differed from the circular shape used in the model. ${ }^{10}$ Furthermore, the applied asynchronous activation of the electrode pads leads to a different behavior than synchronous activation, which needs to be analyzed in the future.

In Experiment 1), it took $3 \mathrm{~min}$ to test the VE at each element and each gap between elements, which is a 

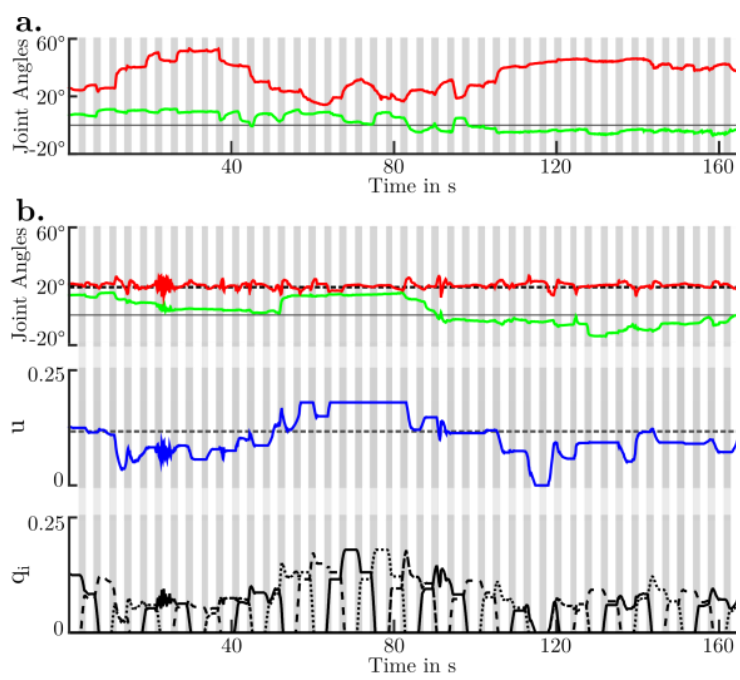

$\cdots r \quad-\alpha \quad-\beta \quad-u_{b} \quad \cdots u_{a} \quad \frac{\cdots}{-} q_{i}$

Fig 4. Results for Experiment 1) with $d=12 \mathrm{~mm}$ : a. open-loop with a constant stimulation intensity of $u_{a}=0.12 ; \boldsymbol{b}$. closed-loop with the controlled intensity $u_{b}$ in blue. The VE center $\boldsymbol{p}$ was moved from one element to the next during the gray zones. The red and green lines are the measured wrist angles, the thin black line marks an angle of $0^{\circ}$. The bottom plot depicts the output of the interpolation block: the stimulation intensity $q_{i}$ on each element depending on $u_{b}$ and the position $\boldsymbol{p}$ of the VE.

worst-case scenario. We suppose that in a clinial setup, the therapist would only search in an area of the array where he/she assumes to evoke the desired motion. Therefore, the time for the search will be significantly reduced. We were further able to reduce the setup time of our framework by just recording step responses at five elements which were spread across the array.

In summary, we presented a feedback-control-assisted manual search strategy which enables the therapist to conveniently modify a VE within an array for a desired motion. The results of four healthy volunteers for hand extension demonstrated the feasibility of our approach. In the future, we are planning to establish a tablet as a visualization and input device for our method. The therapist will be enabled to move the VE in a visualized array by his/her finger. Number, size, and shape of the VEs will also be adjustable.

\section{Contributions}

CS, method development, data collecting, data analyzing, and manuscript writing; MV, design of the electrode array and stimulation system; TSch, method development, and data analyzing; all authors, manuscript reviewing.
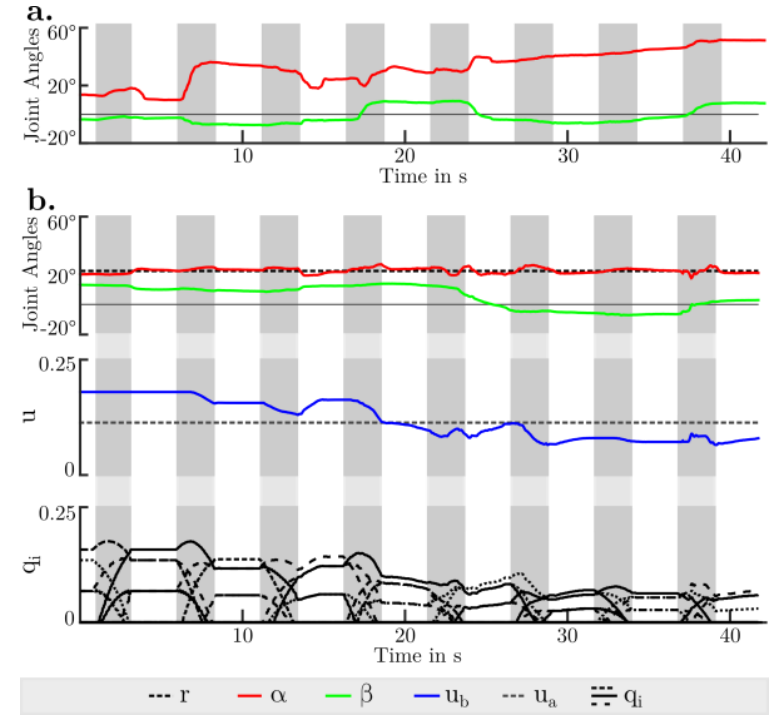

Fig 5. Results for Experiment 2) with $d=30 \mathrm{~mm}$ : $\boldsymbol{a}$. open-loop with a constant stimulation intensity of $u_{a}=0.11 ; \boldsymbol{b}$. closed-loop with the controlled intensity $u_{b}$ in blue. The VE center $\boldsymbol{p}$ was changed during the gray zones; white zones symbolize the stimulation at the positions of the arrowheads in Fig. 3.b.

\section{Acknowledgements}

The work was conducted within the research project BeMobil, which is supported by the German Federal Ministry of Education and Research (BMBF) (FKZ16SV7069K).

\section{Conflict of Interest}

The authors declare no potential conflict of interests.

\section{Corresponding Author}

Christina Salchow, Technische Universität Berlin, Fakultät für Elektrotechnik und Informatik, Fachgebiet Regelungssysteme, Sek. EN 11, Einsteinufer 17, 10587 Berlin, Germany - www.control.tu-berlin.de E-mail: salchow@ control.tu-berlin.de E-mails of Authors

Markus Valtin - valtin@control-tu-berlin.de Thomas Seel - seel@control.tu-berlin.de Thomas Schauer - schauer@control.tu-berlin.de

\section{References}

1. Keller T, Lawrence M, Kuhn, Morari M. New multi-channel transcutaneous electrical stimulation technology for rehabilitation. In Conf Proc IEEE Eng Med Biol Soc, 2006;194-7. doi: 10.1109/IEMBS.2006.259399 


\section{Finding suitable virtual electrodes in arrays using an interpolation strategy}

Eur J Transl Myol 26 (2) 134-139

2. Popović D, Popović M. Automatic determination of the optimal shape of a surface electrode: selective stimulation. Journal of Neuroscience Methods, 2009;178:174-81. doi: 10.1016/j.jneumeth.2008. 12.003

3. O'Dwyer S, O'Keeffe D, Coote S, Lyons G. An electrode configuration technique using an electrode matrix arrangement for FES-based upper arm rehabilitation systems. Med Eng Phys, 2006; 28:166-76. doi: 10.1016/j.medengphy.2005.03.010

4. Hoffmann U, Deinhofer M, Keller T. Automatic determination of parameters for multipad functional electrical stimulation: Application to hand opening and closing. In Conf Proc IEEE Eng Med Biol Soc, 2012;1859-63. doi: 10.1109/EMBC.2012.6346314

5. Malešević N, Maneski L, Ilić V, Jorgovanović N, Bijelić G, Keller T, Popović D. A multi-pad electrode based functional electrical stimulation system for restoration of grasp. J Neuroeng Rehabil 2012;9:66. doi: 10.1186/1743-0003-9-66.

6. Valtin M, Seel T, Raisch J, Schauer T. Iterative learning control of drop foot stimulation with array electrodes for selective muscle activation. In
Preprints of the 19th World Congress, The International Federation of Automatic Control, Cape Town, South Africa, 2014;6586-92. doi: 10.3182/20140824-6-ZA-1003.01991

7. Åström K, Hägglund T. PID controllers: theory, design, and tuning. 2nd ed. USA: Instrument Society of America; 1995. 343 p.

8. Valtin M, Schauer T, Behling C, Daniel M, Weber M. New multi-channel transcutaneous electrical stimulation technology for rehabilitation. In Proc Biodevices, 2012;345-9. doi: 10.1109/IEMBS.2006.259399

9. Shalaby R. Development of an electromyography detection system for the control of functional electrical stimulation in neurological rehabilitation [dissertation]. [Berlin]: Technische Universität Berlin; 2011. 135 p. doi: 10.14279/depositonce-2904

10. Keller T, Kuhn A. Electrodes for transcutaneous (surface) electrical stimulation. Journal of Automatic Control 2008;18:35-45. doi: 10.2298/JAC0802035K 